\title{
Efficacy of low- and moderate-intensity statins for achieving low- density lipoprotein cholesterol targets in Thai type 2 diabetic patients
}

Nuntakorn Thongtang ${ }^{*}$ (D), Chaiyut Sitthananun, Sutin Sriussadaporn and Wannee Nitiyanant

\begin{abstract}
Background: Low dose statins are commonly used among Asians, because plasma low-density lipoprotein cholesterol (LDL-C) reductions similar to those observed in Westerners are achieved at lower doses. We aimed to assess the efficacy of low- and moderate-intensity statins for achieving plasma lipid targets in Thai type2 diabetes (T2D) and to evaluate factors associated with greater LDL-C reduction by statins.

Methods: T2D patients who were treated with low- and moderate-intensity statins at the Siriraj Diabetes Clinic during the January 2013 to December 2014 study period were eligible for inclusion( $n=978), 400$ patients were randomly recruited. Patients were classified into 1 of the following 2 groups according to their plasma LDL-C reductions by statins $(N=393)$; very favorable response ( $L D L-C$ reduction $\geq 50 \%$ ) or less favorable response ( $L D L-C$ reduction $<50 \%)$.

Results: Of the 400 patients, $41.3 \%$ were low-intensity statin users. Mean age was $64.4 \pm 12.7$ years, $64 \%$ were female. Median duration of diabetes was 13.3 years and mean HbA1C was $8.1 \pm 1.9 \%$. Plasma LDL-C goal of $<100 \mathrm{mg} / \mathrm{dl}$ and $<70 \mathrm{mg} / \mathrm{dl}$ was achieved in $84.3 \%$ and $38.0 \%$ respectively, with no significant difference between the low- and moderate-intensity statin users. LDL-C reductions $\geq 50 \%$ can be achieved in $38.4 \%$. Factors associated with very favorable responses from statins were age, hypertension, patients with stable or reduced weight, and better glycemic control.

Conclusion: Low- and moderate-intensity statins achieved plasma LDL-C goal of $<100 \mathrm{mg} / \mathrm{dl}$ and $<70 \mathrm{mg} / \mathrm{dl}$ in 84 . $3 \%$, and $38.4 \%$ of the patients respectively. Due to the improved response to lower doses observed in Asians, a titration dosage strategy should be considered.
\end{abstract}

Keywords: Statins, Intensity, Diabetes, Asians

\section{Background}

Type 2 diabetes (T2D) is an independent risk factor for atherosclerotic cardiovascular disease (ASCVD) and has been classified as coronary heart disease (CHD) risk equivalent [1-3]. Statin treatment has been shown to be effective in reducing ASCVD events both in primary and secondary prevention $[4,5]$. The American Diabetes Association 2016 guideline [6] and the American College

\footnotetext{
* Correspondence: Nuntakorn@hotmail.com

Division of Endocrinology and Metabolism, Faculty of Medicine Siriraj

Hospital, Mahidol University, 2 Wanglang Road, Bangkoknoi, Bangkok 10700, Thailand
}

of Cardiology/American Heart Association 2013 guideline [7] both recommended using moderate- and highintensity statins in all T2D patients, except those aged $<40$ years without ASCVD risk factors. Low-, moderate-, and high-intensity statins therapy are predicted to reduce plasma low-density lipoprotein cholesterol (LDL-C) levels from baseline by approximately $<30 \%, 30 \%$ to $<50 \%$, and $\geq 50 \%$, respectively [7]. Low-intensity statins are not recommended in these guidelines for patients with T2D $[6,7]$. However, previous data suggested that Asians achieved plasma LDL-C reductions that were similar to those observed in Westerners, except at lower statin doses 
[8-11]. A proposed explanation for this dose-effect difference was genetic variability in drug metabolism [12]. Treatments with simvastatin $10 \mathrm{mg} /$ day and atorvastatin $10 \mathrm{mg} /$ day over 8 weeks at 6 Asian centers resulted in average plasma LDL-C reductions of $35 \%$ and $43 \%$, respectively [10]. In addition, blood statin levels were reported to be higher in Asians than in Westerners at the same statin doses, which suggest an increased risk of statin toxicity in Asian population. Lee, et al. reported a difference in plasma LDL-C reduction response to rosuvastatin between subjects of European and Asian ancestry living in Singapore [13]. Moreover, Asian subjects had approximately twofold greater plasma exposure to rosuvastatin than Caucasians, which was not the result of body weight or environmental factors [13]. Although high-intensity statin therapy has been used effectively and safely in Asian patients especially ASCVD patients, most statin side effects are dose-dependent [14-16]. Titration of statin dosage is a common approach among Thai physicians thus low- and moderate-intensity statins are commonly prescribed among Thai T2D patients without ASCVD.

The aim of this study was to assess the efficacy of lowand moderate-intensity statins for achieving plasma lipid targets in Thai T2D patients, and to assess factors associated with greater plasma LDL-C reductions during statin therapy.

\section{Methods}

This retrospective cohort study was conducted at Siriraj hospital, Mahidol University- Thailand's largest universitybased national tertiary referral center. All of the study subjects are Asians. T2D patients who were treated with low- and moderate-intensity statins at the Siriraj Diabetes Clinic during the January 2013 to December 2014 study period were eligible for inclusion. Patients were stratified into 3 groups by statin intensity according to American College of Cardiology/American Heart Association (ACC/ AHA) 2013 guideline; as low-, moderate-, or high-intensity (Additional file 1). Patients were included if they met the following criteria during the study period: (I) age $\geq 18$ years; (II) diagnosed with T2D; (III) received low- to moderateintensity stable statin doses for at least 2 months; and, (IV) had baseline lipid profile laboratory investigation. The medical records of all subjects were reviewed and demographic and clinical data, including diabetic complications, medications used, and blood chemistry, were recorded. The presence of ASCVD was defined as the presence of CHD, ischemic stroke, peripheral artery disease or aortic aneurysm diagnosed during the data collection. The protocol for this study was approved by the Siriraj Institutional Review Board (SIRB) ethical committee. This research was done in accordance with the Ethical Principles of Medical
Research Involving Human Subjects outlined in the declaration of Helsinki in 1975.

A total of 1,081 patients attended Siriraj Diabetes Clinic during January 2013 to December 2014 and were statin users. Low- and moderate-intensity statins were prescribed in 978 (90.47\%) of the patients while highintensity statins were prescribed in only 103 patents (9.53\%). Of the 978 low- to moderate-intensity statin users and based on the results of the sample size formula shown below, 400 patients were randomly selected by using random sample numbers generating from an Excel program (Fig. 1). Baseline plasma LDL-C level before statin therapy was available in 393 patients. Percentages of plasma LDL-C reduction by statin therapy were calculated using the following formula:

$\left(\frac{(\text { Pre-stain plasma LDL-Clevel-plasma LDL-C at the time of enrollment })}{\text { Pre-statin plasma LDL-C }}\right) \times 100$

Patients were classified into 1 of the following 2 groups according to their percentage plasma LDL-C reduction after statin treatments; very favorable (LDL-C reduction $\geq 50 \%$ ) or less favorable response (LDL-C reduction $<50 \%)$. Factors associated with greater plasma LDL-C reduction were analyzed (Fig. 1).

\section{Sample size calculations}

We estimated that low-and moderate-intensity statins would achieve plasma LDL-C of $<70 \mathrm{mg} / \mathrm{dl}$ in $35 \%$ of the subjects based on our own previous study (unpublished). A sample size of 350 subjects was required to obtain a $95 \%$ confidence interval with a margin error of $5 \%$ according to the formula: $\mathrm{n}=\mathrm{Z}_{\alpha}^{2} \mathrm{P}(1-\mathrm{P}) / \mathrm{d}^{2}$. A sample size was round up to 400 subjects for approximately $14 \%$ sample loss.

Factors associated with greater plasma LDL-C reductions were calculated based on the formula using in the case control study. If baseline LDL $\geq 160 \mathrm{mg} / \mathrm{dl}$ is prevalent $43 \%$ and $23 \%$, respectively in the group with LDL-C reduction $\geq 50 \%$ and $<50 \%$. A sample size of 124 per

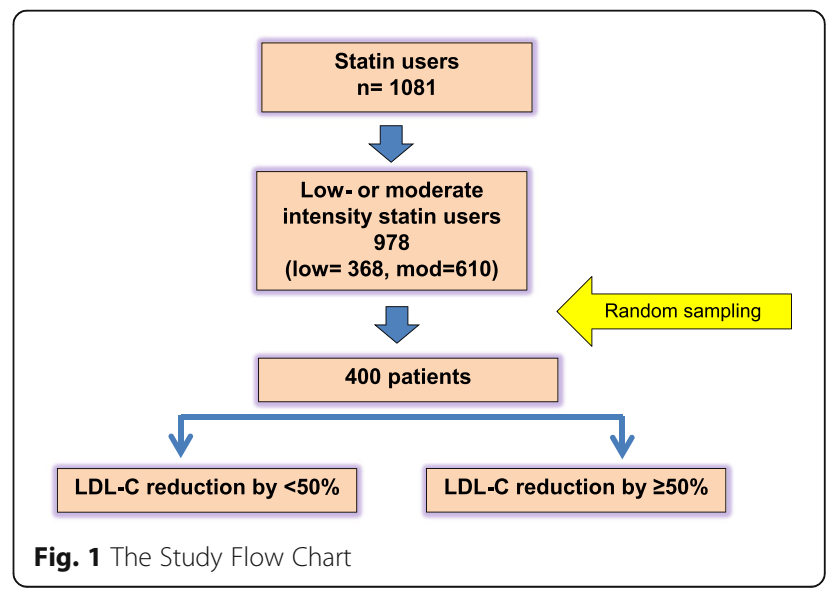


group is required for a ratio of case to control of 1:1 with a $95 \%$ confidence and a power of $90 \%$.

In order to achieve both objectives, the sample size of 400 is used in this study.

\section{Statistical analysis}

All statistical analyses were performed using SPSS software version 20 (SPSS, Inc, Chicago, IL, USA). Categorical variables were compared using Fisher's exact test or chi-square analysis and continuous variables were compared using Student's $t$-test or Mann-Whitney $U$ test. Data are presented as numbers (\%), percentages, mean $\pm \mathrm{SD}$, or median. All statistical tests were 2-tailed. A $p$ value $\leq 0.05$ was considered to be statistically significant.

\section{Results}

\section{Demographic data}

Of 400 patients, 165 patients (41\%) used low-intensity statins and 235 patients (59\%) used moderate-intensity statins. Patient demographic and clinical data are summarized in Table 1. Mean age of patients was $64.4 \pm$ 12.7 years, and $64 \%$ were female. Study participants had a long duration of diabetes, with a median duration of 13.3 years. Mean $\mathrm{HbA1C}$ was $8.1 \pm 1.9 \%$. In most patients, statin drugs had been prescribed for an extended period of time. Median duration of statin use was 63 months. Patients who received moderate-intensity statins had significantly $(p<0.05)$ higher prevalence of ASCVD, CHD, chronic kidney disease (CKD), and higher systolic blood pressure (SBP) than patients who received low-intensity statins. Other clinical characteristics were similar between the low and moderate groups. Chronic diabetic complications were also not significantly different between groups. Pre-statin plasma lipid levels are showed in Table 1. Mean total cholesterol and mean plasma LDL-C were $219.3 \pm 45.6 \mathrm{mg} / \mathrm{dl}$ and 138.9 $\pm 37.6 \mathrm{mg} / \mathrm{dl}$, respectively. Patients who received moderate-intensity statins had significantly higher baseline (pre-statin treatment) plasma cholesterol, triglyceride, and LDL-C levels than patients in the low-intensity statin group. Pre-statin plasma HDL-C levels were also significantly lower in moderate-intensity statin users than in low-intensity statin users.

\section{LDL-C goal achievement}

We found that 337 patients $(84.3 \%)$ who received lowor moderate-intensity statins can achieve plasma LDL-C goal of less than $100 \mathrm{mg} / \mathrm{dl}$, and 152 patients (38\%) achieved LDL-C goal of less than $70 \mathrm{mg} / \mathrm{dl}$ (Fig. 2). Interestingly, moderate-intensity statins did not result in a significant greater LDL-C reduction goals achievement rate than the low-intensity statins. In fact, low-intensity statin users demonstrated a trend toward greater achievement of LDL-C reduction $<70 \mathrm{mg} / \mathrm{dl}$ than moderate-intensity statin users. The percentages of patients achieving plasma LDL-C goals in the low- and moderate-intensity statin groups were $87.3 \%$ vs. $82.1 \%$, respectively for plasma LDL-C goal of $<100 \mathrm{mg} / \mathrm{dl}$, and $43.6 \%$ vs. $34.0 \%$, respectively for LDL-C goal $<70 \mathrm{mg} / \mathrm{dl}$ (Fig. 2).

\section{Percentages of plasma LDL-C reductions by statins}

The median percentage of plasma LDL-C reduction by statins of the overall participants $(\mathrm{N}=393)$ were $-34.6 \%$, while low- and moderate-intensity statin group had median percentages of plasma LDL-C reduction by $-31.4 \%$ and $-36.1 \%$, respectively. Moreover; we found that $75.9 \%$ of low-intensity statin users had plasma LDL-C reduction greater than $30 \%$ from baseline, which is higher than the LDL-C reduction expected in the Westerners. Moreover $41.1 \%$ of moderate-intensity statin users, and $34.5 \%$ of the low-intensity statin users had plasma LDL-C reduction $\geq 50 \%$ from baseline which is expected to achieved from high-intensity statins in the Westerners (Fig. 3). As compared to low-intensity statins, moderate-intensity statins did not result in a greater proportion of patients having percentage baseline LDLC reduction of $\geq 50 \%$ or $30 \%$ to $<50 \%$ than low-intensity statins (Fig. 3).

\section{Factors predicting greater plasma LDL-C reduction with statins}

There were $38.4 \%(n=151)$ of subjects who had plasma LDL-C reduction $\geq 50 \%$ from baseline and this group was defined as the very favorable response group. The remaining participants who had plasma LDL-C reduction $<50 \%$ were classified as the less favorable response group $(n=242)$. Patients in the very favorable response group were significantly older and had longer duration of statin use than the lessfavorable response group. Hypertension was more prevalent in the very favorable response group. Moreover, the very favorable response group had a significantly greater number of patients who could maintain or reduce their body weight after statin therapy was initiated, as compared to the lessfavorable response group (Table 2). Blood chemistry results before statin treatment of both groups are shown in Table 2. Subjects with better glycemic control had greater LDL-C lowering response with low- and moderate-intensity statins than subjects with poorer glycemic controls. Mean baseline HbA1C were significantly lower in the very favorable response group than the less favorable response group $(7.7 \%$ vs. $8.3 \%, p<$ $0.01)$. The very favorable response group had higher mean plasma total cholesterol and LDL-C levels than the less favorable response group (Table 2). 
Table 1 Clinical Characteristics of the Study Participants

\begin{tabular}{|c|c|c|c|c|}
\hline Characteristic & Overall $(n=400)$ & Low-intensity statins $(n=165)$ & Moderate-intensity statins $(n=235)$ & $p$-value \\
\hline Female & $256(64.0 \%)$ & $113(68.5 \%)$ & $143(60.8 \%)$ & 0.12 \\
\hline Age (mean $\pm S D$, years) & $64.4 \pm 12.7$ & $64.0 \pm 13.5$ & $64.7 \pm 12.1$ & 0.62 \\
\hline Duration of DM (median, years) & 13 & 14 & 12 & 0.13 \\
\hline Duration of statins (median, months) ${ }^{a}$ & 63 & 63 & 61.5 & 0.46 \\
\hline Smoking & $10 / 355(2.8 \%)$ & $3 / 146(2.1 \%)$ & $7 / 209$ (3.3\%) & 0.63 \\
\hline Drink alcohol & 28/352 (8.0\%) & $7 / 146(4.8 \%)$ & $21 / 206(10.2 \%)$ & 0.06 \\
\hline Family history of CHD & $5 / 186(2.7 \%)$ & $3 / 83(3.6 \%)$ & $2 / 103(1.9 \%)$ & 0.81 \\
\hline Weight before statin treatment (kg) & $66.6 \pm 13.1$ & $66.8+13.4$ & $66.4+12.9$ & 0.74 \\
\hline Body weight at enrollment (kg) & $67.9 \pm 14.4$ & $66.9 \pm 12.5$ & $68.5 \pm 15.5$ & 0.30 \\
\hline Body mass index $\left(\mathrm{kg} / \mathrm{m}^{2}\right)$ & $26.4 \pm 5.7$ & $26.4 \pm 4.8$ & $26.4 \pm 6.2$ & 0.96 \\
\hline Waist circumference (cm) & $93.5 \pm 11.5$ & $93.0 \pm 11.7$ & $93.9 \pm 11.42$ & 0.72 \\
\hline Systolic blood pressure (mmHg) & $132.7 \pm 14.8$ & $130.7 \pm 15.2$ & $134.1 \pm 14.4$ & 0.02 \\
\hline Diastolic blood pressure (mmHg) & $74.5 \pm 11.0$ & $73.47 \pm 11.2$ & $75.2 \pm 10.9$ & 0.13 \\
\hline \multicolumn{5}{|l|}{ Comorbidity } \\
\hline ASCVD & $79 / 400(19.8 \%)$ & $24 / 165(14.5 \%)$ & $55 / 235(23.4 \%)$ & 0.03 \\
\hline $\mathrm{CHD}$ & $57 / 400(14.3 \%)$ & 16/165 (9.7\%) & $41 / 235(17.4 \%)$ & 0.02 \\
\hline Stroke & $27 / 400(6.8 \%)$ & 9/165 (5.5\%) & 18/235 (7.7\%) & 0.39 \\
\hline Peripheral artery disease & $11 / 400(2.8 \%)$ & $3 / 165(1.8 \%)$ & $8 / 235(3.4 \%)$ & 0.52 \\
\hline Aortic aneurysm & $2 / 400(0.5 \%)$ & 0/165 (0.0\%) & $2 / 235(0.9 \%)$ & 0.64 \\
\hline Hypertension & 296/400 (74.0\%) & $124 / 165(75.2 \%)$ & $172 / 235(73.2 \%)$ & 0.66 \\
\hline Chronic kidney disease & $106 / 400(26.5 \%)$ & $34 / 165(20.6 \%)$ & $72 / 235(30.6 \%)$ & 0.03 \\
\hline Diabetic retinopathy & & & & 0.88 \\
\hline No DR & 232/347 (66.9\%) & $102 / 140(72.9 \%)$ & 130/207 (62.8\%) & \\
\hline NPDR & $83 / 347(23.9 \%)$ & $25 / 140(17.9 \%)$ & $58 / 207(28.0 \%)$ & \\
\hline PDR & $32 / 347(9.2 \%)$ & $13 / 140(9.3 \%)$ & 19/207 (9.2\%) & \\
\hline Diabetic neuropathy & & & & 0.66 \\
\hline Normal & 120/165 (72.7\%) & $45 / 65(69.2 \%)$ & $75 / 100(75.0 \%)$ & \\
\hline Impaired monofilament test & $30 / 165(18.2 \%)$ & $14 / 65(21.5 \%)$ & $16 / 100(16.0 \%)$ & \\
\hline Foot ulcer & 15/165 (9.1\%) & $6 / 65(9.2 \%)$ & $9 / 100(9.0 \%)$ & \\
\hline Diabetic nephropathy & & & & 0.43 \\
\hline MAU $<30$ mg/g creatinine & 175/296 (59.1\%) & 73/118 (61.9\%) & $102 / 178(57.3 \%)$ & \\
\hline MAU $\geq 30 \mathrm{mg} / \mathrm{g}$ creatinine & $121 / 296(40.9 \%)$ & $45 / 118(38.1 \%)$ & $76 / 178(42.7 \%)$ & \\
\hline \multicolumn{5}{|l|}{ Pre-statin plasma lipid levels } \\
\hline Total cholesterol (mg/dl) & $219.3 \pm 45.6$ & $211.9 \pm 40.3$ & $224.6 \pm 48.4$ & 0.01 \\
\hline Triglyceride (mg/dl) & $157.3 \pm 78.7$ & $143.1 \pm 67.8$ & $167.2 \pm 84.3$ & 0.01 \\
\hline $\mathrm{HDL}(\mathrm{mg} / \mathrm{dl})$ & $51.0 \pm 15.1$ & $53.0 \pm 15.5$ & $49.5 \pm 14.8$ & 0.04 \\
\hline LDL-C (mg/dl) & $138.9 \pm 37.6$ & $132 \pm 33.6$ & $143.7 \pm 39.6$ & $<0.01$ \\
\hline
\end{tabular}

Data presented as number (\%), mean $\pm \mathrm{SD}$, or median; $p$-value $<0.05$ indicates statistical significance. ${ }^{\mathrm{a}}$ Duration of statin was counted from the time of statin initiation until the data collection date. Pre-statin plasma LDL-C levels were available in 393 patients

CHD, coronary heart disease; DM, diabetes mellitus; BMI, body mass index; ASCVD, atherosclerotic cardiovascular disease; DR, diabetic retinopathy; NPDR, nonproliferative diabetic retinopathy; PDR, proliferative diabetic retinopathy; MAU, microalbuminuria;. HDL-C, high-density lipoprotein cholesterol; LDL-C, low-density lipoprotein cholesterol

\section{Discussion}

Low- and moderate-intensity statins were commonly used in Thai T2D patients, and were prescribed in $34.04 \%$ and $56.43 \%$, respectively among statin users while high-intensity statins were prescribed in only 9.53\%. We found that low- and moderate-intensity statins can achieve plasma LDL-C reduction goal of $<100$ and $<70 \mathrm{mg} / \mathrm{dl}$ in $84.3 \%$ and $38 \%$ of the patients, 


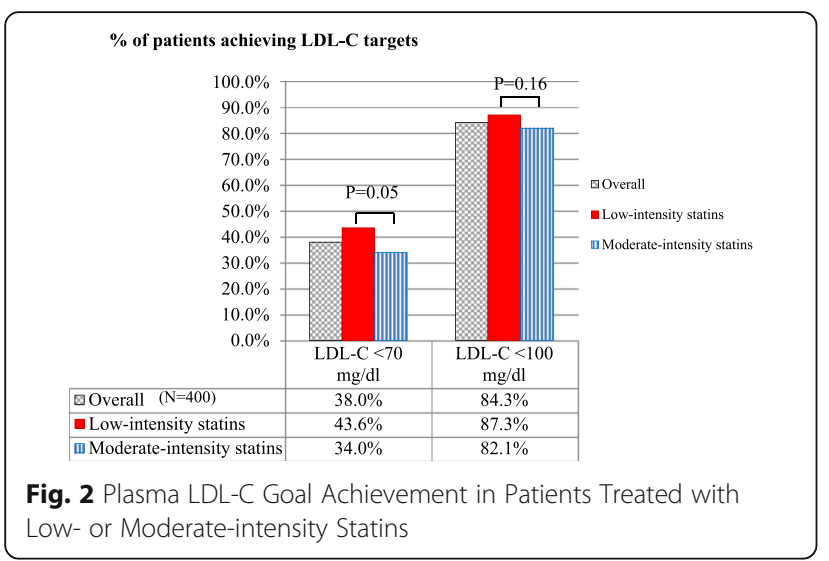

respectively. Moreover low- and moderate-intensity statins resulted in plasma LDL-C reduction $\geq 50 \%$ in $34.5 \%$ and $41.1 \%$ of patients, respectively. In fact, plasma LDL reductions greater than 50\% from baseline are expected to result from high-intensity statin therapy in the Westerners $[7,15]$. Overall our data demonstrated greater efficacy of plasma LDL-C reductions with low doses of statins in Thai diabetic patients, which is consistent with the results of other studies in Japanese [9], Korean [8], and Chinese [10,17] subjects. Matsuzawa Y, et al. found that $5 \mathrm{mg}$ of simvastatin in Asians obtained the same LDL-C reduction effect as $20 \mathrm{mg}$ of simvastatin use in Westerners [9]. A possible explanation for the greater effect of statin at lower doses may center on differences in statin pharmacokinetics and pharmacogenetics between Asian and Western populations resulted in higher blood statin levels in Asians than Westerners with the same statin dosage [11-13, 17, 18].

Interestingly, moderate-intensity statins do not produce greater LDL-C goal achievement rate or greater percentage of baseline LDL-C reduction, as compared with low-intensity statins in Thai diabetic patients. Doubling of statin dosage resulted in greater plasma LDL-C reduction of approximately $6 \%[19,20]$, thus may explain the non-significant increase in LDL-C goal achieving rate in our study. However, a meta-analysis of

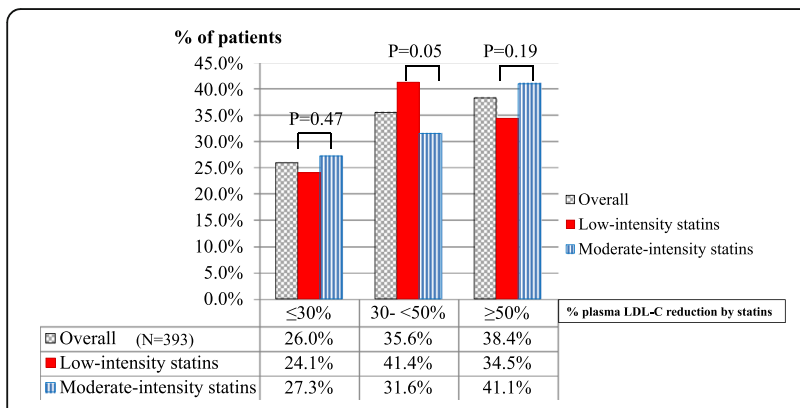

Fig. 3 Percentage of Plasma LDL-C Reduction by Low- and Moderateintensity Statin Therapy statin trials reported greater CAD risk reduction with high-intensity statins as compared to the lower intensity statins [4]. It is still unclear whether this additional benefit is due to the greater LDL-C reduction or from other pleiotropic effects of statins. Moderate-potency statins were more commonly used than low-potency statins in our clinic, thus moderate-potency statin users were randomly recruited more into our study. The presence of ASCVD, the presence of cardiovascular risk factors such as hypertension, chronic kidney disease, and higher pre-statin plasma lipid levels were associated with physicians' choice of using higher potency statins. These are reasonable approach according to the recent guidelines $[6,7]$.

Our study found that factors associated with greater plasma LDL-C reduction in response to low- and moderate-intensity statins were older age, longer duration of statin usage, hypertension, weight maintenance or weight loss during statin therapy, lower HbA1c, and higher baseline plasma lipids. Some of these factors were consistent with the previous studies. Con, et al. found that the probability of attaining the LDL-C goal increases with age [21]. Trompe, et al. studied characteristics of non-responders and high responders of pravastatin treatment among the elderly and found that patients who did not respond well to pravastatin therapy were, on average, 1 year younger [22]. Higher blood statin level in the elderly might be another explanation for the greater plasma LDL-C reduction response. The finding of longer duration of statin usages in the very favorable LDL-C reduction response group may also reflect the longer duration of hypercholesterolemia in the older patients. In addition, the ability to maintain body weight or lose weight predicts a better LDL-C lowering response during statin treatment. We speculate that the ability to maintain body weight or lose weight results from an improved self-perception of one's health status. These patients may have healthier lifestyles and may engage in regular exercise in addition to statin treatment, so these patients may have better response to statin therapy. Moreover, a better glycemic control was another predictor of better LDL-C lowering response during statin therapy. This also supports the concept of better self-care in this group.

\section{Limitation}

This study has some limitations. First, this was a retrospective study and plasma lipid levels were recorded only at baseline before statins initiation and at the last two visits. As such, biochemical changes between times and between changes in medications were not investigated. Second, we only evaluated low- and moderateintensity statins as they were most commonly prescribed in Thailand for primary prevention of ASCVD; 
Table 2 Demographic and Clinical Characteristics of Patients by LDL-C Reduction Group ( $<50 \%$ vs. $\geq 50 \%)$

\begin{tabular}{|c|c|c|c|}
\hline & LDL-C reduction $<50 \%(n=242)$ & LDL-C reduction $\geq 50 \%(n=151)$ & $p$-value \\
\hline Female & $152(62.8 \%)$ & $99(65.6 \%)$ & 0.58 \\
\hline Age (years) & $63.2 \pm 13$ & $66.4 \pm 12.1$ & 0.02 \\
\hline Duration of DM (mo, median) & 13 & 13 & 0.29 \\
\hline Duration of statin usage (mo, median) & 56 & 88 & $<0.01$ \\
\hline Smoking & $7 / 215(3.3 \%)$ & $3 / 133(2.3 \%)$ & 0.86 \\
\hline Alcohol & $17 / 213(8.0 \%)$ & 10/132 (7.6\%) & 0.89 \\
\hline Family history of $\mathrm{CHD}$ & $1 / 112(0.9 \%)$ & $4 / 72(5.6 \%)$ & 0.08 \\
\hline Body mass index $\left(\mathrm{kg} / \mathrm{m}^{2}\right)$ & $26.2 \pm 4.9$ & $26.6 \pm 6.8$ & 0.58 \\
\hline Weight before statin treatment $(\mathrm{kg})$ & $65.9 \pm 12.6$ & $67.4 \pm 13.9$ & 0.28 \\
\hline Waist circumference (cm) & $94.6 \pm 11.9$ & $91.2 \pm 10.4$ & 0.19 \\
\hline Weight reduction & $87 / 227(38.3 \%)$ & 68/138 (49.3\%) & 0.04 \\
\hline $\mathrm{SBP}(\mathrm{mmHg})$ & $131.8 \pm 14.3$ & $74.9 \pm 11.3$ & 0.11 \\
\hline $\mathrm{DBP}(\mathrm{mmHg})$ & $74.9 \pm 11.3$ & $73.8 \pm 10.8$ & 0.33 \\
\hline \multicolumn{4}{|l|}{ Comorbidities } \\
\hline $\mathrm{CHD}$ & $35 / 242(14.5 \%)$ & 20/151 (13.2\%) & 0.74 \\
\hline Stroke & 18/242 (7.4\%) & 9/151 (6.0\%) & 0.57 \\
\hline Peripheral artery disease & $6 / 242(2.5 \%)$ & $5 / 151(3.3 \%)$ & 0.63 \\
\hline Aortic aneurysm & $2 / 242(0.8 \%)$ & $0 / 151(0.0 \%)$ & 0.26 \\
\hline Hypertension & $167 / 242(69.0 \%)$ & $124 / 151(82.0 \%)$ & $<0.01$ \\
\hline Chronic kidney disease & $65 / 242(26.9 \%)$ & $40 / 151(26.5 \%)$ & 0.94 \\
\hline Diabetic retinopathy & & & 0.79 \\
\hline Normal & $135 / 206(65.5 \%)$ & $94 / 136(69.1 \%)$ & \\
\hline NPDR & $51 / 206(24.8 \%)$ & $30 / 136(22.1 \%)$ & \\
\hline PDR & 20/206 (9.7\%) & $12 / 136(8.8 \%)$ & \\
\hline Diabetic neuropathy & & & 0.75 \\
\hline Normal & 75/105 (71.4\%) & $43 / 58(74.1 \%)$ & \\
\hline Impaired Monofilament & $21 / 105$ (20.0\%) & $9 / 58(15.5 \%)$ & \\
\hline Foot ulcer & 9/105 (8.6\%) & $6 / 58(10.4 \%)$ & \\
\hline Diabetic nephropathy & & & 0.93 \\
\hline MAU $\geq 30 \mathrm{mg} / \mathrm{g}$ creatinine & $71 / 175(40.6 \%)$ & 48/117 (41.0\%) & \\
\hline $\mathrm{MAU}<30 \mathrm{mg} / \mathrm{g}$ creatinine & 104/175 (59.4\%) & $69 / 117(59.0 \%)$ & \\
\hline Statin potency & & & 0.19 \\
\hline Low-intensity statin users & $106 / 242(43.8 \%)$ & $56 / 151(37.1 \%)$ & \\
\hline Moderate-intensity statin users & $136 / 242(56.2 \%)$ & $95 / 151(62.9 \%)$ & \\
\hline \multicolumn{4}{|l|}{ Pre-statin blood chemistry } \\
\hline Total cholesterol (mg/dl) & $204.7 \pm 43.8$ & $242.4 \pm 39.1$ & $<0.01$ \\
\hline Triglyceride (mg/dl) & $153.5 \pm 81.0$ & $161.8 \pm 74.6$ & 0.35 \\
\hline $\mathrm{HDL}(\mathrm{mg} / \mathrm{dl})$ & $50.9 \pm 16.3$ & $51.1 \pm 13.1$ & 0.95 \\
\hline LDL-C (mg/dl) & $125.7 \pm 34.1$ & $160.0 \pm 33.2$ & $<0.01$ \\
\hline $\mathrm{HbA} 1 \mathrm{C}(\%)$ & $8.3 \pm 2.0$ & $7.7 \pm 1.6$ & $<0.01$ \\
\hline FBS (mg/dl, median) & 158.9 & 169.4 & 0.32 \\
\hline
\end{tabular}

\footnotetext{
Data presented as mean \pm SD or median; $p$-value $<0.05$ indicates statistical significance
}

$C H D$, coronary heart disease; $D M$, diabetes mellitus; $B M I$, body mass index; $A S C V D$, atherosclerotic cardiovascular disease; $D R$, diabetic retinopathy; NPDR, nonproliferative diabetic retinopathy; $P D R$, proliferative diabetic retinopathy; $M A U$, microalbuminuria; $L D L-C$, low-density lipoprotein cholesterol; SD, standard deviation; HDL-C, high-density lipoprotein cholesterol; FBS, fasting blood sugar 
therefore, our data cannot be applied to high-intensity statins. Third, only $19.8 \%$ of our study population had ASCVD. As such, our findings can be applied mainly to T2D for primary prevention, but cannot be generalized for use in secondary prevention. Moreover, in a realworld setting, inter-individual variability in statin responsiveness may be more evident $[23,24]$.

\section{Conclusions}

Thai diabetic patients primarily receive low- or moderate-intensity statins. Low- and moderate-intensity statins achieved the plasma LDL-C reduction goal of $<100$ and $<70 \mathrm{mg} / \mathrm{dl}$ in $84.3 \%$ and $38 \%$ of the patients, respectively. Predictors of better response to statin therapy were older age, stable or reduced weight, and better glycemic control. Due to the improved response to lower doses observed in Asians, a titration dosage strategy should be considered.

\section{Additional file}

Additional file 1: Statin intensity classification according to American College of Cardiology/American Heart Association (ACC/AHA) 2013 guideline. (PPTX $63 \mathrm{~kb}$ )

\section{Abbreviations}

ASCVD: Atherosclerotic cardiovascular disease; CKD: Chronic kidney disease; CHD: Coronary heart disease; LDL-C: Low-density lipoprotein cholesterol; SBP: Systolic blood pressure; T2D: Type2 diabetes

\section{Acknowledgements}

Not applicable

\section{Funding}

This study received research funding from Siriraj Research Grant.

\section{Availability of data and materials}

All data supporting our findings are within the paper.

\section{Authors' contributions}

NT designed the study. CS gathered the clinical data. NT, CS analyzed the data. NT, SS wrote the main paper. All authors discussed the results and commented on the manuscript at all stages. All authors read and approved the final manuscript.

\section{Competing interest}

The authors declare that they have no competing interests.

\section{Consent for publication}

Not applicable.

\section{Ethics approval and consent to participate}

This study was approved by the Siriraj Institutional Review Board (SIRB) ethical committee.

Received: 16 November 2016 Accepted: 7 February 2017 Published online: 13 February 2017

\section{References}

1. Haffner SM, Lehto S, Ronnemaa T, Pyorala K, Laakso M. Mortality from coronary heart disease in subjects with type 2 diabetes and in nondiabetic subjects with and without prior myocardial infarction. N Engl J Med. 1998; 339(4):229-34
2. Bragg F, Li L, Yang L, Guo Y, Chen Y, Bian Z, et al. Risks and population burden of cardiovascular diseases associated with diabetes in china: a prospective study of 0.5 million adults. PLoS Med. 2016; 13(7):e1002026.

3. Daniels LB, Grady D, Mosca L, Collins P, Mitlak BH, Amewou-Atisso $M G$, et al. Is diabetes mellitus a heart disease equivalent in women? results from an international study of postmenopausal women in the raloxifene use for the heart (RUTH) trial. Circ Cardiovasc Qual Outcomes. 2013;6(2):164-70.

4. Cholesterol Treatment Trialists C, Baigent C, Blackwell L, Emberson J, Holland LE, Reith C, et al. Efficacy and safety of more intensive lowering of LDL cholesterol: a meta-analysis of data from 170,000 participants in 26 randomised trials. Lancet. 2010;376(9753):1670-81.

5. Cholesterol Treatment Trialists C, Kearney PM, Blackwell L, Collins R, Keech A, Simes J, et al. Efficacy of cholesterol-lowering therapy in 18,686 people with diabetes in 14 randomised trials of statins: a meta-analysis. Lancet. 2008;371(9607):117-25.

6. American Diabetes Association. Standards of Medical Care in Diabetes-2016: Summary of Revisions. Diabetes Care. 2016;39(Suppl 1):S4-5.

7. Stone NJ, Robinson JG, Lichtenstein AH, Bairey Merz CN, Blum CB, Eckel RH, et al. 2013 ACC/AHA guideline on the treatment of blood cholesterol to reduce atherosclerotic cardiovascular risk in adults: a report of the American College of Cardiology/American Heart Association Task Force on Practice Guidelines. Circulation. 2014;129(25 Suppl 2):S1-45.

8. Jee Eun Kwon YK, Hyun S, Won H, Shin S, Lee K, Sang-Wook Kim THK, Kim CJ. Cholesterol Lowering Effects of Low-dose Statins in Korean Patients. J Lipid Atheroscle. 2014;3(1):21-8.

9. Matsuzawa Y, Kita T, Mabuchi H, Matsuzaki M, Nakaya N, Oikawa S, et al. Sustained reduction of serum cholesterol in low-dose 6-year simvastatin treatment with minimum side effects in 51,321 Japanese hypercholesterolemic patients. Circ J. 2003;67(4):287-94.

10. Wu CC, Sy R, Tanphaichitr V, Hin AT, Suyono S, Lee YT. Comparing the efficacy and safety of atorvastatin and simvastatin in Asians with elevated low-density lipoprotein-cholesterol-a multinational, multicenter, doubleblind study. J Formos Med Assoc. 2002;101(7):478-87.

11. Liao JK. Safety and efficacy of statins in Asians. Am J Cardiol. 2007;99(3):410-4.

12. Wang P. Statin dose in Asians: is pharmacogenetics relevant? Pharmacogenomics. 2011;12(11):1605-15.

13. Lee E, Ryan S, Birmingham B, Zalikowski J, March R, Ambrose $H$, et al. Rosuvastatin pharmacokinetics and pharmacogenetics in white and Asian subjects residing in the same environment. Clin Pharmacol Ther. 2005:78(4):330-41.

14. Jacobson TA. NLA Task Force on Statin Safety-2014 update. J Clin Lipidol. 2014;8(3 Suppl):S1-4.

15. Thongtang N, Ai M, Otokozawa S, Himbergen TV, Asztalos BF, Nakajima K, et al. Effects of maximal atorvastatin and rosuvastatin treatment on markers of glucose homeostasis and inflammation. Am J Cardiol. 2011;107(3):387-92.

16. Study of the Effectiveness of Additional Reductions in C, Homocysteine Collaborative G, Armitage J, Bowman L, Wallendszus K, Bulbulia R, et al. Intensive lowering of LDL cholesterol with $80 \mathrm{mg}$ versus $20 \mathrm{mg}$ simvastatin daily in 12,064 survivors of myocardial infarction: a double-blind randomised trial. Lancet. 2010;376(9753): 1658-69.

17. Hu M, Lui SS, Ko GT, Tomlinson B. Do the lipid responses to rosuvastatin and atorvastatin differ between Chinese and Caucasians? Comparison of the DISCOVERY-Hong Kong study with other DISCOVERY studies. Int J Cardiol. 2013;168(3):3071-3.

18. Lee HK, Hu M, Lui S, Ho CS, Wong CK, Tomlinson B. Effects of polymorphisms in ABCG2, SLCO1B1, SLC10A1 and CYP2C9/19 on plasma concentrations of rosuvastatin and lipid response in Chinese patients. Pharmacogenomics. 2013;14(11):1283-94.

19. Nicholls SJ, Brandrup-Wognsen G, Palmer M, Barter PJ. Meta-analysis of comparative efficacy of increasing dose of Atorvastatin versus Rosuvastatin versus Simvastatin on lowering levels of atherogenic lipids (from VOYAGER). Am J Cardiol. 2010;105(1):69-76.

20. Zhao SP, Yu BL, Peng DQ, Huo Y. The effect of moderate-dose versus double-dose statins on patients with acute coronary syndrome in China: Results of the CHILLAS trial. Atherosclerosis. 2014;233(2):707-12.

21. Cone C, Murata G, Myers O. Demographic determinants of response to statin medications. Am J Health Syst Pharm. 2011;68(6):511-7. 
22. Trompet S, Postmus I, Slagboom PE, Heijmans BT, Smit RA, Maier AB, et al. Non-response to (statin) therapy: the importance of distinguishing nonresponders from non-adherers in pharmacogenetic studies. Eur J Clin Pharmacol. 2016;72(4):431-7.

23. Postmus I, Trompet S, Deshmukh HA, Barnes MR, Li X, Warren HR, et al. Pharmacogenetic meta-analysis of genome-wide association studies of LDL cholesterol response to statins. Nat Commun. 2014;5:5068.

24. Postmus I, Verschuren JJ, de Craen AJ, Slagboom PE, Westendorp RG, Jukema JW, et al. Pharmacogenetics of statins: achievements, wholegenome analyses and future perspectives. Pharmacogenomics. 2012;13(7):831-40

Submit your next manuscript to BioMed Central and we will help you at every step:

- We accept pre-submission inquiries

- Our selector tool helps you to find the most relevant journal

- We provide round the clock customer support

- Convenient online submission

- Thorough peer review

- Inclusion in PubMed and all major indexing services

- Maximum visibility for your research

Submit your manuscript at www.biomedcentral.com/submit
Biomed Central 